\title{
Criterios de calidad en el diseño pedagógico de un curso virtual
}

\author{
Nora Astrid Corredor Gómez ${ }^{1}$
}

\begin{abstract}
Resumen
La investigación indaga sobre los criterios de calidad en el diseño pedagógico en cursos virtuales se centra en el análisis de la motivación y los estilos de aprendizaje y su relación con los recursos de aprendizaje seleccionados para el desarrollo de los cursos virtuales. Se utilizaron dos instrumentos para la recolección de datos la observación y la entrevista. Se analizaron los resultados a través del diseño de investigación de teoría fundamentada que permitió estudiar las categorías y sub categorías a través de la codificación abierta y las relaciones entre categorías a través de la codificación axial. Finalmente se obtuvo el esquema teórico, estableciendo los criterios de calidad reflejados en estrategias de motivación para un curso virtual, considerando los intereses y preferencias de los estudiantes a través de los estilos de aprendizaje y la interacción entre estudiantes y profesores, al ofrecer diversos recursos y actividades de aprendizaje. Estas estrategias se proponen para ser implementadas en la fase de diseño y el desarrollo de un curso virtual.
\end{abstract}

Palabras clave: ambiente educacional, aprendizaje en línea, interés (aprendizaje), medio de aprendizaje, proceso de aprendizaje. 


\title{
Quality criteria for instructional design of an online course
}

\begin{abstract}
This study aims to assess the quality criteria in designing virtual courses. Is focus on the motivation analysis and learning styles and their relationship to selected learning resources for developing online courses. Two instruments were used for data collection, observation and interview. The results were analyzed through the grounded theory research design that allowed study categories and sub categories through open coding and relationships between categories through axial coding. Finally, we obtained the theoretical framework establishing quality criteria reflected in motivational strategies for a virtual course, taking in account students interests and preferences through learning styles and interaction between students and teachers, offering various resources and learning activities. These strategies are proposed to be implemented in the design and development phase of an online course.
\end{abstract}

Key words: E-learning, educational environment, interest (learning), learning environment, learning process.

Recibido: 1 de abril de 2013 Aceptado: 24 de mayo de 2013

\section{Introducción}

La educación virtual en Colombia en los últimos años ha crecido en el número de programas, cursos y estudiantes, siendo esta una oportunidad de educación para personas con diversas características socioeconómicas, para quienes sus ocupaciones laborales y el lugar donde viven resultan ser factores limitantes para acceder a la educación presencial. En este sentido establecer criterio de calidad de un programa de un curso virtual resulta fundamental. 
Al analizar la situación de la educación superior en el contexto colombiano se encontró que los estándares de calidad específicos para la educación virtual están en proceso de elaboración, actualmente existe una propuesta inicial que refleja las preocupaciones y los resultados observados de diferentes programas de educación virtual (MEN, 2010).

Otra característica que se encontró en Colombia fue la preocupación alrededor del alto índice de deserción, de 47\% para el periodo 2002-2007, según el estudio realizado por el Ministerio de Educación Nacional (2009), entre los determinantes de la deserción se encontraron: expectativas no satisfechas con el programa, los recursos de apoyo, la interacción con los profesores, el apoyo académico y psicológico y los recursos económicos para continuar, frente a esta situación las universidades han fortalecido los programas de bienestar con el fin de generar mayor satisfacción del estudiante en su entorno universitario.

La pregunta de investigación se planteó con el fin de determinar los criterios de calidad de los cursos virtuales que consideren las características personales de los estudiantes y que incluyan estrategias de motivación, que promuevan en los estudiantes la continuación de sus estudios hasta su finalización. Para lograr la comprensión de este tema se determinó la pregunta: ¿Cuáles criterios de calidad en el diseño pedagógico de un curso virtual se relacionan con los estilos de aprendizaje y la motivación en estudiantes universitarios?. Analizando tres variables durante la investigación: los estilos de aprendizaje, la motivación y los recursos de aprendizaje.

Durante la investigación se consideró una definición de calidad directamente relacionada con la educación virtual. Seoane y García (2006) definen la calidad en e-learning como: "la efectiva adquisición de una serie de competencias, habilidades, conocimientos y destrezas por parte de un conjunto de alumnos, mediante el desarrollo de contenidos de aprendizaje adecuados, impartidos a través de unas herramientas web eficientes y con el apoyo de una red de servicios añadidos, cuyo proceso está garantizado por un exhaustivo y personalizado proceso de evaluación y certificación, y monitorizado por un equipo humano que ejerce una labor tutorial integral durante todo el proceso de enseñanza-aprendizaje". De esta forma el diseño de cursos virtuales con estándares de calidad implica tener en cuenta diversas variables que permiten lograr la efectividad del curso, medida en el éxito del aprendizaje de los estudiantes. 


\section{Marco teórico}

En el desarrollo del marco teórico se realizó una revisión documental en los estudios de calidad en educación virtual, la motivación, los estilos de aprendizaje y los recursos educativos.

En relación a la calidad de los cursos virtuales se realizó la revisión de investigaciones realizadas centrando la atención en el análisis del componente pedagógico. Restrepo (2005) destaca la interacción constante entre tutores y estudiantes, la realimentación inmediata, la dosificación de lecturas y actividades, el aprendizaje por descubrimiento y construcción; el aprendizaje cooperativo y el énfasis en estrategias de aprendizaje. Santoveña (2005) considera los materiales disponibles, las características de los contenidos didácticos, las herramientas de contenidos, de comunicación y la motivación como factores de calidad. En el estudio preliminar el Ministerio de Educación Nacional (2010), se encuentran: actividades sincrónicas limitadas, considerar el tiempo de las actividades, autonomía en el aprendizaje, el diseño de estrategias para el desarrollo de competencias.

En el tema de motivación se estudió la motivación intrínseca y extrínseca para vincular estas estrategias en los recursos educativos específicamente en la educación virtual. Se estudió la posición de Vigotsky (2007) donde se considera que la motivación para aprender se determina por los valores que sostienen y justifican el aprendizaje (¿para qué aprender?), Vigotsky señala que las actividades de construcción de nuevos conceptos constituyen la motivación en sí misma y no requiere de refuerzo externo, es decir, una motivación extrínseca. Adicionalmente Fuente (2004) diferencia entre motivación intrínseca y motivación extrínseca, establece que la motivación intrínseca se fundamenta en que el objetivo es lograr aprender y los resultados del aprendizaje son asumidos totalmente por el estudiante mientras que la motivación extrínseca centra la atención del estudiante en los estímulos externos para obtener buenos resultados.

Según Ormrod (2008) para mejorar el ambiente de aprendizaje y promover la motivación de los estudiantes y un buen rendimiento, es importante tener en cuenta que el profesor proporciona un ambiente motivador a través de la motivación extrínseca, satisfaciendo las necesidades de expresión y afecto, generando emociones relacionadas con los temas, evaluando para mejorar y promoviendo la metacognición, y el estudiante utiliza su motivación intrínseca y autorregula sus acciones y sus emociones a favor de aprendizaje.

En el estudio documental acerca de los estilos de aprendizaje se abordaron diferentes clasificaciones que se han realizado considerando: la preferencia por 
las estrategias de aprendizaje la preferencia por los recursos de aprendizaje, las preferencias en el ambiente de aprendizaje, las preferencias en la forma cómo interactúan con otros.

David Kolb (1981), a partir de su Teoría Aprendizaje Experimental, considera que el aprendizaje es cocebido como un ciclo de cuatro niveles: experiencia concreta: al involucrarse en nuevas experiencias, observación reflexiva: cuando observan y reflexionan sobre esas experiencias desde muchas perspectivas, abstracción conceptual: al crear conceptos que integran sus observaciones y experimentación activa: al utilizar esas teorías para tomar decisiones y resolver problemas. Según David Kolb (1981) los aprendizajes, necesitan cuatro diferentes tipos de habilidad para ser efectivos, estilo divergente: prefieren trabajo en grupo, tienen la habilidad para organizar muchas relaciones, estilo asimilativo: su fortaleza está en la habilidad para crear modelos teóricos, estilo convergente: tienen la habilidad para resolver problemas y tomar decisiones, estilo servicial: su fortaleza está en hacer cosas, en llevar a cabo planes y experimentos.

En el modelo VARK (visual, auditivo, lector/escritor, kinestésico) de Neil Fleming (1995), se considera que el éxito académico está relacionado con una técnica de aprendizaje apropiada, afirma que las personas aprenden de diferentes formas y presenta una clasificación de preferencias, auditivos: prefieren la información hablada, lectores/escritores: prefieren acceder a la información desde las palabras impresas, visuales: les gusta la información en forma de gráficos, cuadros, diagramas de flujo, kinestésicos: les gusta aprender utilizando sus sentidos, desean experiencias concretas multisensoriales

En el marco de proporcionar estrategias de aprendizaje que se adapten a cada estilo de aprendizaje, Arias (2009) realiza una propuesta de cursos virtuales adaptativos, en los cuales el proceso de enseñanza-aprendizaje sea planificado y ejecutado de acuerdo a las características de cada estudiante y a su ritmo de estudio de forma individualizada. El proceso busca seleccionar los mejores contenidos educativos y de acuerdo con las características particulares que posee cada estudiante, que permita obtener para cada estudiante el mejor recurso según su estilo de aprendizaje y en las evaluaciones generar preguntas adaptadas a las necesidades de cada estudiante.

A través del análisis de investigaciones realizadas acerca de los estilos de aprendizaje se observa que existen diferentes clasificaciones y en el momento de reconocer los diferentes estilos en los recursos educativos de los cursos virtuales se plantean dos estrategias, la primera es reconocer los estilos de aprendizaje con diferentes estrategias donde todas las habilidades y preferencias son importantes en el proceso de aprendizaje, la segunda es asignar recursos educativos específicos de acuerdo al estilo de aprendizaje de cada estudiante. 
En la revisión documental en relación a los recursos de aprendizaje se identifican las perspectivas de Vigotsky, Piaget y Dale. Vigotsky (2007) resalta la comunicación verbal y también considera posibles combinaciones entre comunicación verbal y visual para favorecer y estimular los procesos constructivos. Piaget (2001) señala que las funciones esenciales de la inteligencia son la comprensión y la invención, explica que la sensación, la imagen y la asociación juegan un papel importante. A través del cono de experiencia, Edgar Dale (1964) identifica diferentes niveles de experiencias de aprendizaje, considera que una práctica de aprendizaje puede abarcar desde la experiencia directa (concreta) hasta el uso de los símbolos visuales y verbales (abstracto) en el momento de estudiar un determinado tema, considerando en su modelo tanto los recursos de aprendizaje como las actividades de aprendizaje.

Para el análisis de los recursos educativos se realizó una clasificación en tres grupos: recursos textuales, audiovisuales e interactivos, la investigación se centró en la relación con la motivación y los estilos de aprendizaje de los estudiantes y la inclusión de estas dos variables en el diseño y selección de recursos de aprendizaje para un curso virtual.

Bryndum y Monte (2005) consideran que los integrantes de las comunidades de aprendizaje en línea pueden realizar diferentes acciones en los espacios de interacción, permitiendo la multivariedad de posturas frente a la diversidad de sus integrantes, existen diversas tareas posibles para que este proceso individual de cada alumno lo conduzca a estar motivado dentro de un programa virtual. La multimedia favorece la presentación de las tareas en diferentes formas, porque presenta los contenidos desde diferentes sesgos, respetando los diferentes estilos de aprendizaje para abordar la información.

\section{Metodología}

El proceso de investigación realizado aplica la metodología cualitativa a partir de la cual fue posible relacionar las estrategias de motivación y los estilos de aprendizaje en los recursos educativos utilizados en los cursos virtuales, para conocer con profundidad la relación entre las variables se utilizaron dos técnicas de recolección de datos la observación y la entrevista. Las entrevistas permitieron tener en cuenta las perspectivas de los estudiantes y profesores vinculados a los cursos virtuales y la observación de cursos virtuales permitió conocer la estructura de los cursos y contenidos por parte del investigador. Para el análisis de datos se seleccionó el diseño de investigación de teoría fundamentada. Para la validación de datos se realizó la triangulación de los resultados obtenidos a partir de la aplicación de los instrumentos relacionando la información desde diferentes fuentes. 
En la Tabla 1 se observan los puntos de referencia para exponer la relación entre las variables (motivación, estilos de aprendizaje y recursos de aprendizaje), las preguntas que se incluyeron en los instrumentos de investigación (observación $\mathrm{y}$ entrevistas) y las fuentes de información (estudiante, profesores y la plataforma virtual).

\begin{tabular}{|c|c|c|c|}
\hline Fuentes & Plataforma virtual & Estudiantes & Profesores \\
\hline Instrumento & Observación & Entrevista & Entrevista \\
\hline \multicolumn{4}{|c|}{ Variables } \\
\hline \multicolumn{4}{|l|}{ Motivación } \\
\hline $\begin{array}{l}\text { Motivación intrínseca y } \\
\text { extrínseca }\end{array}$ & $\begin{array}{l}\text { ¿Qué tipo de estrategias de } \\
\text { motivación se ofrecen en la } \\
\text { estructura del curso? } \\
\text { ¿Las estrategias de } \\
\text { motivación son continuas } \\
\text { durante el desarrollo del } \\
\text { curso? }\end{array}$ & $\begin{array}{l}\text { ¿Cuáles son los elementos } \\
\text { del curso virtual que lo } \\
\text { motivan para continuar y } \\
\text { terminar el curso virtual? } \\
\text { ¿Cuáles son los elementos } \\
\text { que hacen falta en el } \\
\text { curso para incrementar su } \\
\text { motivación? }\end{array}$ & $\begin{array}{l}\text { ¿Qué tipo de estrategias } \\
\text { emplea para motivar a los } \\
\text { estudiantes? } \\
\text { ¿Cuál de estas } \\
\text { estrategias ha tenido } \\
\text { mejores resultados entre } \\
\text { los estudiantes? }\end{array}$ \\
\hline \multicolumn{4}{|l|}{ Estilos de aprendizaje } \\
\hline $\begin{array}{l}\text { Reconocimiento de los } \\
\text { estilos de aprendizaje. }\end{array}$ & $\begin{array}{l}\text { ¿Las actividades } \\
\text { propuestas vinculan todos } \\
\text { los estilos de aprendizaje de } \\
\text { los estudiantes? }\end{array}$ & $\begin{array}{l}\text { ¿Cuáles son las } \\
\text { actividades del curso que } \\
\text { le han permitido lograr el } \\
\text { aprendizaje? }\end{array}$ & $\begin{array}{l}\text { ¿Cuáles son las } \\
\text { características del } \\
\text { estudiante que tiene en } \\
\text { cuenta en el momento de } \\
\text { diseñar y desarrollar un } \\
\text { curso virtual? }\end{array}$ \\
\hline $\begin{array}{l}\text { Actividades y estilos de } \\
\text { aprendizaje }\end{array}$ & $\begin{array}{l}\text { ¿Cómo se relacionan las } \\
\text { actividades propuestas con } \\
\text { los estilos de aprendizaje? }\end{array}$ & $\begin{array}{l}\text { ¿Cuáles son las actividades } \\
\text { que prefiere realizar durante } \\
\text { el desarrollo del curso? }\end{array}$ & $\begin{array}{l}\text { ¿En el momento de } \\
\text { diseñar las actividades de } \\
\text { aprendizaje en qué forma } \\
\text { considera las diferentes } \\
\text { preferencias de los } \\
\text { estudiantes y estilos de } \\
\text { aprendizaje? }\end{array}$ \\
\hline \multicolumn{4}{|l|}{ Recursos de aprendizaje } \\
\hline Planeación y plataforma & $\begin{array}{l}\text { ¿Existe una descripción de } \\
\text { las actividades a realizar en } \\
\text { el curso, la metodología y } \\
\text { cronograma? } \\
\text { ¿Cuáles son las } \\
\text { herramientas empleadas } \\
\text { durante el desarrollo del } \\
\text { curso? }\end{array}$ & $\begin{array}{l}\text { ¿Alguna vez no ha } \\
\text { realizado alguna de las } \\
\text { actividades propuestas? } \\
\text { ¿Cuál ha sido la razón? } \\
\text { ¿Cuáles han sido los } \\
\text { inconvenientes con el uso } \\
\text { de las herramientas de la } \\
\text { plataforma presentados } \\
\text { durante el desarrollo del } \\
\text { curso virtual? }\end{array}$ & $\begin{array}{l}\text { ¿Durante el diseño del } \\
\text { curso cual ha sido el } \\
\text { proceso de selección de } \\
\text { las herramientas para el } \\
\text { desarrollo del curso? }\end{array}$ \\
\hline Evaluación de recursos & $\begin{array}{l}\text { Aplicación de Evaluación de } \\
\text { Recursos Textuales } \\
\text { Audiovisuales } \\
\text { e Interactivos }\end{array}$ & $\begin{array}{l}\text { ¿Qué tipo de recursos } \\
\text { de apoyo ofrece el curso } \\
\text { virtual? ¿En qué forma los } \\
\text { recursos que se ofrecen son } \\
\text { significativos en su proceso } \\
\text { de aprendizaje? }\end{array}$ & $\begin{array}{l}\text { ¿Cuáles son los recursos } \\
\text { de apoyo (textual, } \\
\text { audiovisual, interactivo) y } \\
\text { en qué forma garantizan } \\
\text { el aprendizaje del } \\
\text { estudiante? }\end{array}$ \\
\hline
\end{tabular}

Tabla 1. Relación entre categorías, instrumentos y fuentes de información (El Autor) 


\section{Análisis de resultados}

En el marco del enfoque cualitativo para el análisis de los resultados se seleccionó el diseño de investigación de la teoría fundamentada, el cual permite establecer una o varias relaciones de asociación entre un fenómeno y sus determinantes (Campo-Redondo y Labarca, 2009). Se realizaron dos procesos para el análisis de los resultados, inicialmente la codificación abierta, donde los segmentos de los datos recolectados se analizan para generar categorías y sub categorías; luego se realiza la codificación axial, donde se organizan las categorías resultantes en un modelo de interrelaciones, reflejando relaciones causales, consecuencias, estrategias, contextos; la visualización de las interrelaciones representa una teoría emergente y explica el fenómeno de estudio, conlleva a la formulación de una teoría sustantiva permitiendo el entendimiento de un fenómeno específico en un ámbito local (Hernández, Fernández y Baptista, 2006).

\section{Codificación Abierta}

El proceso de codificación abierta para el análisis de la información recolectada permitió realizar un esquema de categorías y sub-categorías entre los resultados encontrados a través de los instrumentos de investigación. Las categorías se definieron en concordancia con las variables de estudio, motivación, estilos de aprendizaje y recursos de aprendizaje.

\section{Motivación}

En relación con la motivación, se encontró como una condición inicial del estudiante al seleccionar el programa de formación y la metodología de educación en este caso la modalidad virtual, constituía un elemento de motivación intrínseca para realizar las actividades del curso. Uno de los profesores entrevistados afirmó "quien decide realizar un programa de educación superior ha seleccionado previamente un área de conocimiento especifica de acuerdo a sus intereses personales y esto constituye en sí mismo un elemento de motivación"

En relación a la motivación extrínseca, se observó que la comunicación continua entre profesor y estudiantes resulta ser un elemento de motivación, así mismo cuando los estudiantes reciben retroalimentación frente a los resultados de las actividades, cuando se obtienen respuestas oportunas a las preguntas y cuando se publican continuamente anuncios recordando y explicando detalles para la entrega de actividades. 
Según Fuente (2004) en los procesos de motivación extrínseca los estudiantes centran la atención en los resultados finales de las actividades, depende de estímulos externos para obtener buenos resultados, en este sentido los estímulos externos y recompensas estaría centrados en la retroalimentación y los resultados de las actividades.

Se encontró que considerar los estímulos externos implica tener en cuenta la comunicación y la interacción continua como una estrategia de motivación para realizar las actividades del curso. Uno de los profesores entrevistados describía como estrategia de motivación: "la intermediación de conflictos durante el trabajo en equipos y el seguimiento a los estudiantes a partir de las largas ausencias en su participación en la plataforma”.

Según Ormrod (2008) el profesor puede manifestar su interés y emoción por la materia, generando experiencias positivas, pero a partir de la investigación realizada se pudo determinar que no solamente es la expresión de apreciaciones positivos sino también tener en cuenta la comunicación y la interacción continua entre estudiantes y profesores la estrategia de motivación para realizar las actividades del curso, cuando se rompen los canales de comunicación o las respuestas demoran días los estudiantes tienden a perder el interés en el desarrollo de las actividades del curso.

Desde la perspectiva de los estudiantes la temática y los contenidos del curso resultan ser un factor importante en la motivación, así como el diseño del curso en relación la página y la metodología. Los estudiantes afirmaban "La temática de evaluación es muy importante en el proceso de enseñanzaaprendizaje", "Considero que los elementos del curso virtual que me motivan para continuar y terminar el curso virtual son: el diseño de la página del curso, el programa, las interacciones entre el tutor y los estudiantes, la parte metodológica y pedagógica que presenta el curso, el contenido de la asignatura y su exposición en el diseño del curso virtual”.

En relación a las actividades de aprendizaje, se encontró que la variedad de recursos (adicionales a los textos), tales como recursos audiovisuales, interactivos, las actividades de aplicación del conocimiento, las temáticas de interés para los estudiantes, constituían un conjunto de estrategias de motivación extrínseca.

\section{Estilos de Aprendizaje}

Se encontró que los profesores consideran que el reconocimiento de los diferentes estilos de aprendizaje puede llegar a ser un proceso importante desde el inicio del programa del curso, si bien se ofrecen diferentes tipos de actividades no se realiza un proceso reconocimiento de los estilos de aprendizaje a través de inventarios de clasificación que los diferentes han 
construido a partir de sus modelos de clasificación. Los profesores afirmaban que "actualmente no se identifican los estilos de aprendizaje a través de un proceso formal como la aplicación de test o inventarios", "las actividades dentro de la programación del curso vinculan diferentes tipos de actividades individuales grupales, de reflexión, de análisis, de praxis y teoría."

En cuanto a la vinculación de los estilos de aprendizaje los estudiantes entrevistados manifestaron diferentes preferencias en relación a los recursos de aprendizaje (textual, audiovisual e interactivo), manifestando diferentes preferencias en la forma como se accede a la información. También se encontró entre los estudiantes diferentes preferencias al realizar cierto tipo de actividades de aprendizaje, algunos manifestaron estar cómodos con todas las actividades propuestas, pues consideraban todas importantes para su formación, otros manifestaron preferir las actividades de aplicación práctica de la teoría y otros manifestaron su preferencia por las actividades de discusión en grupos. Uno de los estudiantes afirmaba "siempre realizo todas las actividades porque considero que esto me permite lograr el aprendizaje que necesito y que se deben hacer todas".

En relación a la preferencia por la forma de trabajo, algunos estudiantes manifestaron sentirse cómodos trabajando en grupo y otros consideraban que el trabajo en grupo no se realiza un trabajo eficiente en cuanto el trabajo tiende a realizarlo una sola persona, por lo tanto preferían el trabajo individual. Al respecto los estudiantes entrevistados comentaban "Considero que todas las actividades son importantes, pero me gustan más las individuales porque en grupo, a veces, los compañeros no son tan responsables y comprometidos como uno espera que lo sean y termina uno haciendo todo y los demás ganándose la nota a costas del trabajo y el esfuerzo de otros", "La actividad grupal, permite conjugar la experiencia de todos los integrantes para darle forma al trabajo final del proyecto, creo que es lo más importante de este curso, el trabajo verdaderamente en equipo".

Los estudiantes expresaron satisfacción en el sentido de poder realizar aplicaciones a los conceptos aprendidos, manifestaron que la utilidad del curso era importante en cuanto les permitiera aplicar los conocimientos en su entorno, utilizando el conocimiento de forma práctica. Los estudiantes entrevistados afirmaban "llevar a la práctica lo que se realiza en el curso, como hacerlo más vivencial en nuestra práctica", "De las actividades propuestas, la aplicación directa [...] permite tener claridad de cuales con las diferentes fases y procedimientos que permiten tener un buen proyecto".

Se observó que los cursos impartidos por las universidades consideran todos los estilos de aprendizaje en relación a las preferencias en las actividades y en relación a las preferencias en la forma de trabajo. Los cursos ofrecían diversas actividades, a través de la metodología de aprendizaje por proyectos, a partir de la cual se 
propone que durante el desarrollo del curso se realicen actividades individuales y grupales que tienen en cuenta la comprensión de conceptos que paso a paso van aplicando para el desarrollo de un proyecto, experimentando diversos procesos y desarrollando un ciclo para el aprendizaje en el marco de los estilos de aprendizaje propuesto por Kolb (2007).

Ninguno de los cursos virtuales observados contemplaba todos los tipos de recursos de aprendizaje, se enfocaban principalmente en los recursos textuales, de esta forma no se contemplaban todos los estilos de aprendizaje en relación a la clasificación de Fleming (1995).

Así mismo es importante considerar los estilos de aprendizaje en el diseño de recursos de aprendizaje, ésta consideración permite mantener el interés de los estudiantes, al presentar la información fundamental, las teorías y conceptos básicos de diferentes formas reconociendo las diferentes preferencias de los estudiantes como parte de la motivación extrínseca del proceso de aprendizaje (Fuente, 2004). Se hace evidente la relación directa entre motivación y aprendizaje, por lo cual la motivación debe ser tenida en cuenta en el momento de diseñar experiencias de aprendizaje para alcanzar los objetivos

\section{Recursos de aprendizaje}

Durante el proceso de evaluación de los recursos de aprendizaje ofrecidos por los cursos virtuales, se encontró que los recursos textuales son los más importantes, estos resultan ser la base fundamental de las teorías y los conceptos fundamentales. Uno de los estudiantes entrevistados afirmó: "Para el desarrollo del curso creo que hace falta una mejor interactividad en la diferentes herramientas que se ofrecen para desarrollar las actividades ya que los materiales del curso son solo lecturas".

Se observó que en ocasiones los textos cuentan con otros recursos de aprendizaje que sirven de complemento a la información tales como recursos auditivos y audiovisuales, que contienen elementos explicativos de las teorías expuestas en los textos.

Se observó que ninguno de los cursos ofrece recursos interactivos donde el estudiante revise los conceptos o pueda acceder directamente a un tema determinado. De esta forma ningún curso integraba todos los tipos de recursos para responder a los diferentes estilos de aprendizaje en relación a la forma como se presenta a la información.

Si bien es posible relacionar esta oferta limitada de recursos con la edad del estudiante y el nivel de estudio que imparte, algunos estudiantes entrevistados manifestaron que no era suficiente cuando el curso solamente ofrecía recursos textuales, documentos, lecturas. Uno de los profesores entrevistados afirmó: 
"el nivel educativo del estudiante es importante para decidir acerca de la complejidad de las actividades, asi utilizar un lenguaje especializado de acuerdo al área de conocimiento".

Se encontró que algunos estudiantes manifestaban que el material audiovisual resultaba ser un problema por el limitado acceso a internet en algunas regiones en Colombia. Según afirmaba uno de los estudiantes entrevistados "la principal piedra u obstáculo que he tenido es el acceso a internet con buena velocidad... de hecho para poder observar videos muchas veces me toca madrugar, para encontrar más velocidad...pero en términos generales no he tenido problemas con las herramientas de la plataforma...".

Durante el análisis de los resultados se encontró que la posibilidad de tener acceso a una amplia gama de recursos de aprendizaje (textuales, audiovisuales interactivos), plantea una estrategia integral. Los estudiantes afirmaban: "En los recursos ofrecidos son fáciles de comprender, están diseñados para dar claridad en el tema que pretenden desarrollar", "El curso de excel me pareció muy bueno, tiene lo elemental para manejarlo en forma adecuada, los videos han sido muy buenos e interesantes... y los textos para leer, han sido todos muy buenos...".

También se observó la subutilización de algunas herramientas tecnológicas que ofrecían las plataformas de los cursos virtuales, tales como el wiki, correo, chat, cuya utilización permitirían ofrecer una amplia gama de actividades para los estudiantes.

Durante la investigación se observó que existe una relación importante entre la motivación de los estudiantes, los estilos de aprendizaje y los recursos de aprendizaje. Reconocer la diversidad del estudiante, implica reconocer sus diferentes estilos de aprendizaje al seleccionar diferentes recursos de aprendizaje que considere las diferentes preferencias de los estudiantes. Este proceso permite un elemento más para incrementar la motivación del estudiante y para alcanzar los objetivos de aprendizaje de forma satisfactoria (Bryndum y Monte, 2005).

\section{Codificación axial}

En el proceso de estructurar una respuesta a la pregunta de investigación y partiendo de la condición inicial en la cual se establece la calidad en educación virtual en función del aprendizaje, se identificó la relación entre las categorías estudiadas: motivación, estilos de aprendizaje y recursos de aprendizaje, estableciendo las estrategias que en su conjunto permiten la motivación del estudiante y de esta forma lograr el aprendizaje. 
La información recolectada a partir de los instrumentos permitió realizar un análisis de la relación entre las variables identificando los resultados más significativos para elaborar un esquema teórico de la teoría sustantiva que se estableció como resultado de la investigación (Ver Figura 1).

Los resultados se organizaron de tal forma que el punto central es la motivación, que puede ser intrínseca o extrínseca. La motivación intrínseca se evidencia a partir de la decisión del estudiante por realizar un proceso de formación, desde su iniciativa selecciona el programa de desea estudiar y la modalidad de educación entre educación presencial y a distancia -virtual, este factor de decisión conlleva a estar motivado para realizar todas las actividades y lograr el aprendizaje.

En la motivación extrínseca, el reconocimiento de los estilos de aprendizaje conforma un conjunto de estrategias que fomentan la motivación del estudiante, estas dependen del diseño y desarrollo del curso con el fin de reconocer la diversidad en las preferencias de los estudiantes. Se identificaron tres clasificaciones, las preferencias por las actividades de aprendizaje, las preferencias por la forma como se presenta la información y las preferencias por la forma de trabajo.

Reconocer las preferencias por las actividades atendiendo a los estilos de aprendizaje (convergente, divergente, asimilativo y servicial), implica proponer actividades de observación, experimentación, teorización, aplicación de conocimiento y estrategias de aprendizaje por proyectos donde se involucran los diferentes estilos de aprendizaje. Esto permite mantener el interés del estudiante en las actividades que se realizan.

Las preferencias en la forma como se presenta la información, se tienen en cuenta al ofrecer diversos recursos textuales, audiovisuales e interactivos que generan satisfacción en los estudiantes porque pueden acceder a la información de acuerdo a sus preferencias. Reconocer las habilidades y preferencias en la forma de trabajo, individual y grupal, implica ofrecer a los estudiantes actividades colaborativas de interacción con los compañeros y el profesor así como también actividades individuales, como una estrategia integral.

En este punto también es importante la interacción entre los estudiantes y profesor, porque constituye una estrategia que complementa el conjunto de actividades y recursos de aprendizaje que se proponen en el desarrollo del curso y también constituye una estrategia de motivación extrínseca que se promueve a través de la retroalimentación, las respuestas a preguntas de forma oportuna y la publicación de resultados y valoraciones, permiten garantizar la comunicación continua. 
Criterios de calidad en el diseño pedagógico de un curso virtual. Artículo original producto de la investigación.

La motivación intrínseca y las estrategias de motivación extrínseca que implican el reconocimiento de los estilos de aprendizaje, constituyen un conjunto de estrategias para la motivación que contribuye al éxito en el aprendizaje del estudiante y de esta forma constituyen criterios de la calidad del curso virtual. De esta manera, se establecen criterios de calidad reconociendo las características de cada estudiante a partir de los estilos de aprendizaje, en el diseño de las actividades y recursos de aprendizaje para mantener la motivación del estudiante y lograr los objetivos de aprendizaje propuestos en el curso virtual.

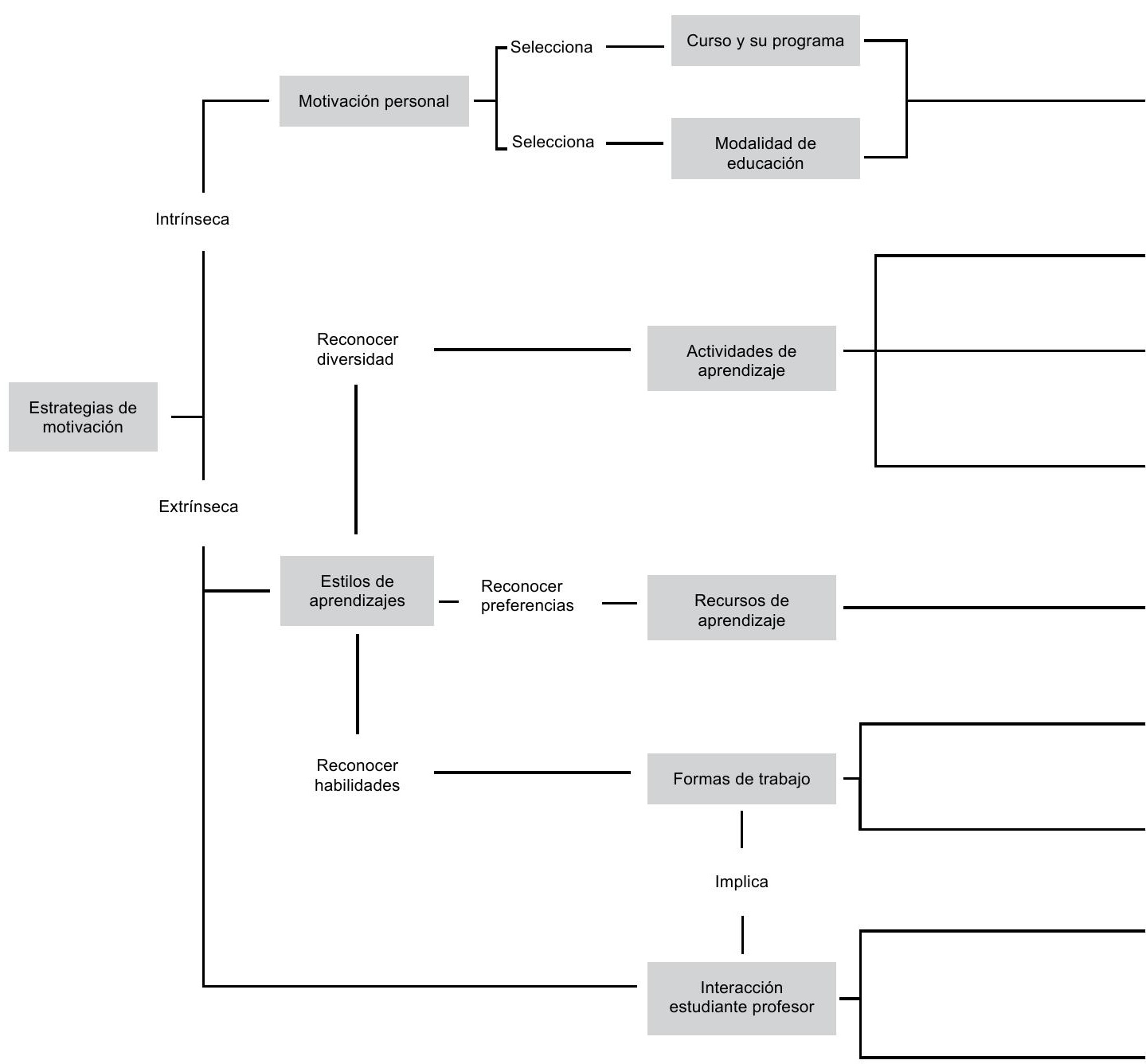




\section{Conclusiones}

En Colombia se encuentra en proceso la definición de los criterios de calidad en educación virtual, los criterios cuentan con diversos aspectos a tener en cuenta para garantizar las características de un programa, en este sentido la investigación realizada ha pretendido abordar una parte de estas características específicamente en relación al diseño pedagógico centrándose en las estrategias definidas para implementar durante el desarrollo del curso.

Frente al tema de deserción en educación superior, para lo cual se han implementado estrategias que son paralelas a las actividades académicas. Se considera la posibilidad de utilizar estrategias que se implementen desde el interior de los cursos, en el diseño de las actividades académicas que pueden llegar a incluir elementos de motivación y reconocimiento de los estilos de aprendizaje de los estudiantes, seleccionando recursos de aprendizaje adecuados que contribuyan con este fin.

Conlleva a
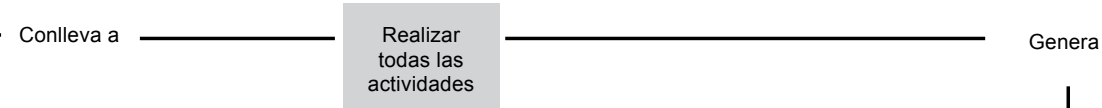

Aplicación del

conocimiento

Aprendizaje por
proyectos

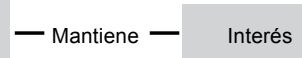

Convergente

Divergente

Asimilativo

Servicial

Textuales

Audiovisuales Interactivos

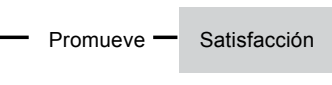

Motivación

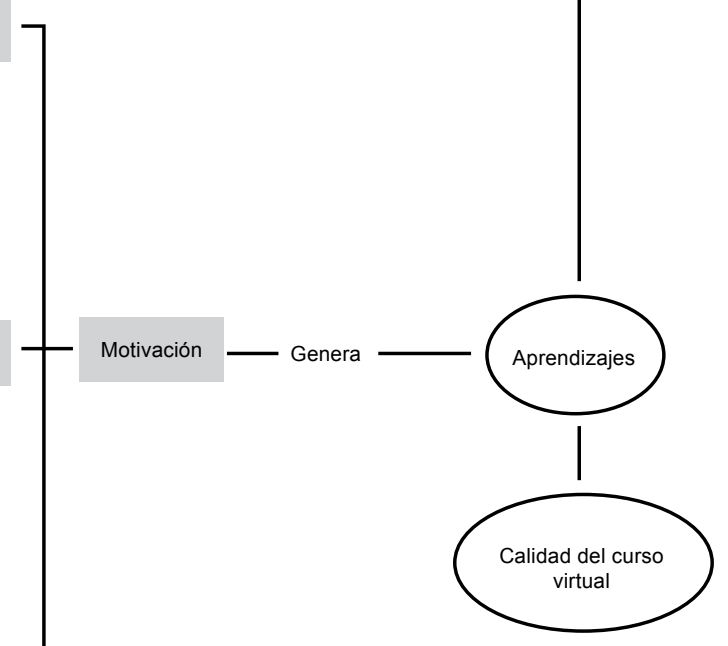

Aprendizajes

Individual

Grupal

Retroalimentación

Figura 1. Esquema teórico (El autor) 
La investigación permitió establecer un conjunto de estrategias para implementar durante la fase de diseño y desarrollo de los cursos virtuales en el marco de los criterios de calidad, inicialmente estrategias de motivación, estrategias de vinculación de los estilos de aprendizaje a través de la selección de recursos de aprendizaje y diseño de actividades de aprendizaje, criterios que en su conjunto contribuyen a que los estudiantes realicen el curso de forma satisfactoria para reducir los niveles de deserción escolar reconociendo a la persona y sus preferencias.

A partir del desarrollo de investigación se destacan diferentes estrategias para aplicar en el diseño pedagógico de un curso virtual:

- Fomentar la interacción estudiante - profesor a través de la comunicación continua, relacionada con la solución de dudas académicas, la retroalimentación a las actividades realizadas por los estudiantes, los anuncios semanales de las actividades a desarrollar y las observaciones para mejorar los resultados.

- Reconocer los estilos de aprendizaje de los estudiantes al seleccionar las actividades de aprendizaje del curso a través de trabajos grupales, individuales, actividades de experimentación, observación, reflexión, análisis, comprobación teórica. Seleccionar actividades de aprendizaje que impliquen la utilización del conocimiento, que permitan realizar prácticas y aplicaciones del conocimiento. La estrategia de aprendizaje por proyectos es un ejemplo que involucra diferentes estilos de aprendizaje y adicionalmente involucra la aplicación del conocimiento, contribuyendo de esta forma a la motivación de los estudiantes.

- Reconocer las diferencias en relación a las preferencias de los estudiantes al seleccionar los recursos de aprendizaje, ofreciendo diversos recursos textuales, audiovisuales e interactivos para reconocer los estilos de aprendizaje de los estudiantes, lo recursos en su conjunto se complementan para lograr la comprensión del tema y el aprendizaje de los estudiantes.

De esta forma en el momento de seleccionar y diseñar las actividades y recursos para el desarrollo de un curso virtual es importante considerar todos los estilos aprendizaje, ya que siguiendo a Kolb estos funcionan como un ciclo en el cual se desarrollan diferentes habilidades que permite al estudiante enfrentarse a nuevas experiencias en la vida personal y laboral. 


\section{Revista de}

La investigación presenta limitantes. En relación al tema de estudio la investigación se centró en el análisis del componente pedagógico. En relación con las necesidades y características del estudiante, se deja al margen del estudio la fundamentación teórica en pedagogía, es decir la selección del modelo pedagógico. En cuanto a los resultados de la investigación es preciso aclarar que no constituyen una teoría formal, solamente es una teoría sustantiva, donde se estudia un fenómeno y se establecen relaciones entre categorías frente a sucesos en un entorno local.

A partir de la investigación realizada surgen nuevos temas por explorar y profundizar. En relación a las variables de estudio, establecer las estrategias para fomentar la motivación intrínseca e identificar cuáles son las estrategias de aprendizaje que contemplan de forma conjunta todos los estilos de aprendizaje. En relación a los estándares de calidad, quedan temas pendientes tales como aspectos tecnológicos, recursos humanos (el papel del profesor y estudiante), aspectos de flexibilización, evaluación del aprendizaje. 


\section{Referencias bibliográficas}

Arias, F.J. (2009). Modelo multi-agente para la planificación instruccional y selección de contenidos en cursos virtuales adaptativos (Tesis de Maestría). De la base de datos de la Universidad Nacional de Colombia.

Campo-Redondo, M. y Labarca, C.(2009).La teoría fundamentada en el estudio empírico de las representaciones sociales: un caso sobre el rol orientador del docente. Redalyc Red de revistas cientificas de America Latina, el Caribe, España y Portugal, 25 (60), 41-54.Disponible en: http://redalyc.uaemex.mx/src/inicio/ArtPdfRed.jsp?iCve=31012531004

Dale, E. (1964). Métodos de enseñanza audiovisual. México: Editorial Reverté.

Fleming, N.D. (1995). I'm different; not dumb. Modes of presentation (VARK) in the tertiary classroom. Trabajo presentado en la Annual Conference of the Higher Education and Research Development Society of Australasia.

Fuente, J. (2004). Perspectivas recientes en el estudio de la motivación: La teoría de la orientación de meta. Revista electrónica de Investigación psicoeducativa, 2(1), 35-62. Disponible en: http://www.investigacion-psicopedagogica.org/revista/new/ContadorArticulo. php?26

Giroux, S., y Tremblay, G. (2004) Metodología de las Ciencias Humanas. Distrito Federal, México: Fondo de Cultura Económica.

Hernández, R., Fernández-Collado, C. y Baptista, P. (2006). Metodología de la investigación. ( $4^{\mathrm{a}}$ ed.). México: McGraw Hill.

Kolb, D. (1981). Learning styles and disciplinary differences. En A. W. Chickering (Ed.) The modern American college responding to the new realities of diverse students and a changing society (pp. 232-255). Estados Unidos de América: Jossey Bass.

Kolb, A. y Kolb, D. (2006). Learning Styles and Learning Spaces: A Review of the Multidisciplinary Application of Experiential Learning Theory in Higher Education. En Sims, R., y Sims, S. (Eds), Learning styles and learning: A key to meeting the accountability demands in education (pp. 45-91). Estados Unidos: Nova Publishers.

Lozano Rodríguez, A. (2007). Reconocimiento de los estilos de aprendizaje en cursos ofrecidos en línea. En Lozano, A., y Burgos, V. (Comp.). Tecnología educativa: en un modelo de educación a distancia centrado en la persona (pp. 157-183). Distrito Federal, México: Limusa.

Ministerio de Educación Nacional (2009). Deserción estudiantil en la educación superior colombiana. Disponible en: http://www.mineducacion.gov.co/ sistemasdeinformacion/1735/articles-254702_libro_desercion.pdf 
Ministerio de Educación Nacional (2010). Propuesta de lineamientos para la educación virtual en la educación superior. Bogotá.

Piaget, J. (2001). Psicología y Pedagogía. Barcelona España: Editorial Crítica.

Restrepo, B. (2005). Consideraciones sobre el aseguramiento de la calidad en educación virtual. Disponible en: http://www.colombiaaprende.edu.co/html/mediateca/1607/ articles-86323_archivo.pdf

Romero, L., Salinas, V. y Mortera, F. (2010). Estilos de aprendizaje basados en el modelo de Kolb en la educación virtual. Apertura, 10 (12), 72-85. Disponible en: http://redalyc. uaemex.mx/redalyc/pdf/688/68815138007.pdf

Salter, D., Evans, N. y Forney, D. (2006). A Longitudinal Study of Learning Style Preferences on the Myers-Briggs Type Indicator and Learning Style Inventory. Journal of College Student Development, 47, 2, ProQuest Education Journals.

Santoveña, S. (2005). Criterios de calidad para la evaluación de cursos virtuales. Revista Eticanet, 2 (4), 18-36. Disponible en: http://tecnologiaedu.us.es/cuestionario/ bibliovir/calidad.pdf

Seoane, A. y García, F. (2006). Criterios de calidad en formación continua basada en eLearning. Una propuesta metodológica de tutoría on-line. Universidad de Salamanca.

Tenant, M. (2005). Transforming Selves. Journal of Transformative Education, 3: 102. Disponible en: http://jtd.sagepub.com/content/3/2/102.

Vigotsky, L., Leontiev, A. y Luria, A. (2007). Psicología y Pedagogía (3 $3^{\mathrm{a}}$ ed.). Madrid España: Ediciones Akal.

Villar, G. (2008). La evaluación de un curso virtual, Propuesta de un modelo. Disponible en: http://www.oei.es/tic/villar.pdf.

Zapalska, A. y Brozik, D, (2007). Learning styles and online Education. Campus-Wide Information Systems, 24 (1), 6-16. Disponible en: www.emeraldinsight.com/1065-0741. htm

Zapata, M. y Albert, M. (2008). Estrategias de aprendizaje y eLearning. Un apunte para la fundamentación del diseño educativo en los entornos virtuales de aprendizaje. RED Revista de Educación a Distancia, 8 (19). Disponible en: http://www.um.es/ead/red/19/ esteban_zapata.pdf 


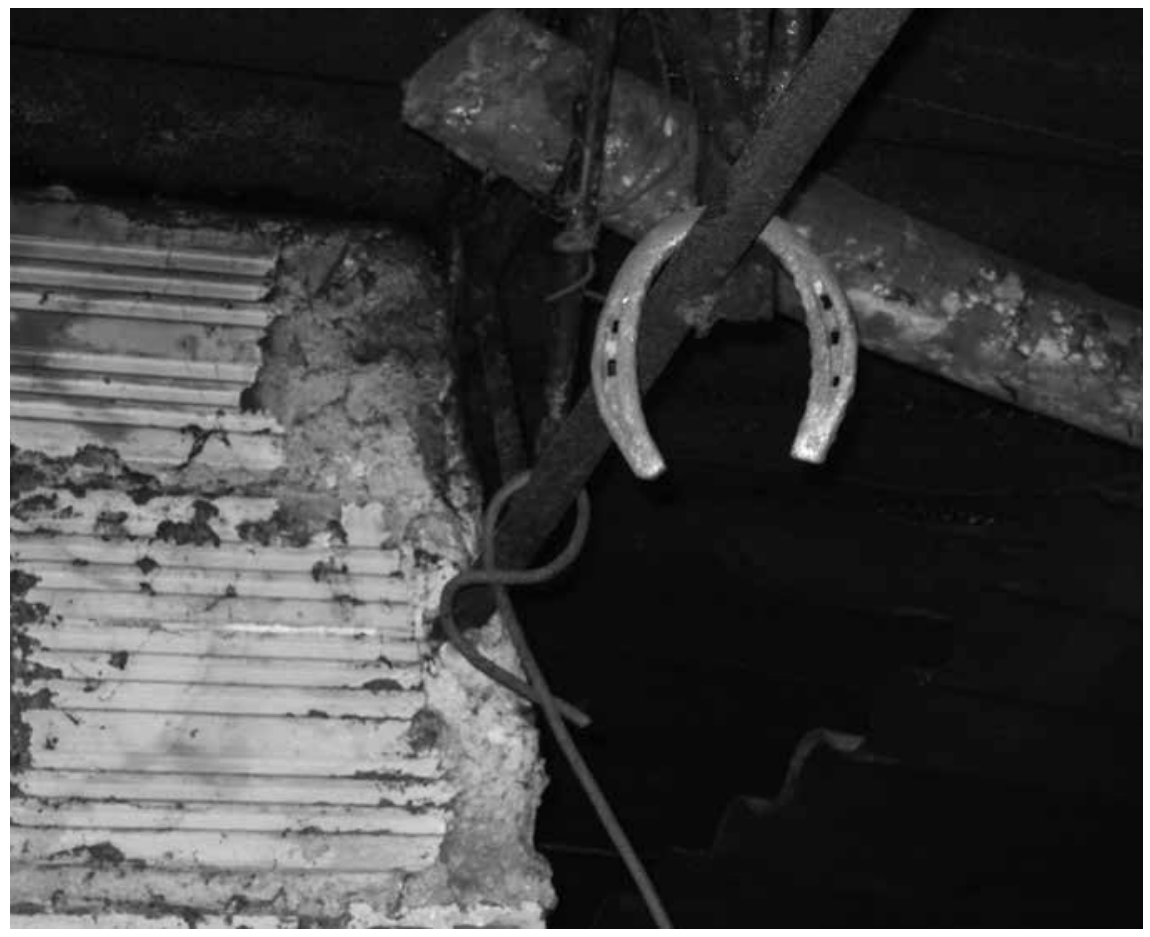

Fotografia: José Enrique Castillo 